\title{
Research on Dynamic Simulation and Optimization of Hospital Medical Process
}

\author{
Wang Quanyu, Wang Yahao, Jia Jinmiao, Liu Xin \\ School of Computer Science \\ Beijing Institute of Technology \\ Beijing 100081
}

\begin{abstract}
The hospital medical treatment process can be modeled as a queuing problem. If the allocation of medical devices and resources is not well optimized, then the passing rate will be low and patients have to be waiting in the queues for a long time. This paper analyzes the hospital medical process and proposes a simulation model based on the queuing theory. An optimization strategy is also proposed to improve the passing rate. According to the simulation results, the optimization significantly reduces the patients' waiting time and increases the passing rate.
\end{abstract}

Keywords-Medical process simulation, Optimization, Queuing theory

\section{INTRODUCTION}

The hospital medical treatment process can be modeled as a queuing problem[1]. Non-optimized allocation of resources may result in unbalanced workload distribution among the entire medical staff, some being overloaded and some being underloaded. This can lead to a very low hospital overall efficiency. The research objective in this problem is to increase the passing rate and reduce the waiting time.

Previous research work can be divided into two classes, patient flow analysis and computer simulation. The patient flow analysis method analyzes all patient treatment process records and the time utilization of all staff in a given time period, trying to identify the bottlenecks in the dynamic "treatment" process that block the patient going forward and prolong the waiting time. The system efficiency will be improved when the bottlenecks are removed[2]. This method is of low cost and it is easy to carry out. However, it is a case-specific method, i.e. each analysis is only suitable for a specific case. And it is infeasible to compare different cases due to the differences, such as the hospital type, class, geographical conditions and economic levels.

The computer simulation method is mainly based on queuing theory. It builds the model of patients reaching the hospital, simulates the medical treatment process of patients, and optimizes the process of hospital care by reengineering of treatment processes and other methods[3]. This method does not make the concrete implementation of the simulation process. But the optimization process requires a lot of manual work to calculate and compare the simulation results, which will lead to a high cost.

In this paper, we design and implement a simulation and optimization software for the hospital medical process based on the queuing theory. The software simulates the process of hospital treatment process and establishes the criteria of resource optimization evaluation. An optimization strategy is also designed so that the patient waiting time in the queue is reduced and the number of hospital patients who get treated is increased.

\section{THE SYSTEM MODEL AND OPTIMIZATION PRINCIPLES}

\section{A. The Model}

This system builds a simulation model based on the standard $\mathrm{M} / \mathrm{M} / 1$ queuing model. A queuing system usually consists of three components: the input process, queuing rules and services [4]. M/M/1 model includes a single desk, infinite customers, a negative exponential distribution for the arrival pattern, and a limited system service time[5]. This system encapsulates medical devices and resources and health care as services desk that processes arrival patients. The three components are as follows:

(i) the input process

The customer consecutively arrives, being independent of each other. The arrival number in a certain time interval obeys a certain probability distribution (Uniform distribution, Negative exponential distribution, Poisson distribution, Normal distribution, Erlang distribution), and the arrival process is stationary.

(ii) Queuing rules

The system has a single waiting queue with unlimited length. The queue follows the first come first serve principle.

(iii) services

There exists a single desk service. The customer service time is independent of each other, following an exponential distribution.

\section{B. The Evaluation Criteria}

Busy ratio $P$ : busy ratio of medical staff is defined as follow:

$$
P=T_{0} / T
$$

where $T_{0}$ is the admission time, $T$ is the working time.

If busy ratio of medical staff is too low, it will cause the waste of medical resources.

Queue length $L$ : queue length is the number of patients in the waiting queue. If the length is too long, patient's satisfaction will decline.

So the objective is to increase the busy ratio $P$ and reduce the queue length $L$ so that the average waiting time for each patient is reduced and the number of hospital patients treated is increased. 


\section{The Optimization Strategy}

The optimization of this system follows the feedback control priciples from automatic control theory. In a feedback control system, the controlling equipment performs control actions on the controlled equipment based on feedback information of controlled variables. The system constantly revises the deviation between controlled variables and controlling variables in order to reach the control target.

Optimation in this system is an iterative feedback control system to the simulation process. The optimization process of the evaluation system, based on evaluation of the system, constantly adjusts the configuration of medical resources in different medical items. It simulates medical treatment processes again after the configuration has been adjusted, and then compares the simulation results. This feedback controlling process is iterated to achieve the best configutation of medical resources.

\section{SySTEM DESIGN AND IMPLEMENTATION}

\section{A. The overall design of system}

Based on the queuing theory and optimization theory of medical resources, this system is divided into four major modules, including patients generated module, medical resource allocation module, simulation module, and optimization module, as shown in Figure 2. The patient generated module implements the input process of queuing theory. Bulk of patients arrive at the hospital for treatment. The number of each batch arrival is a random variable, and arrival interval between batches subjects to specific probability distribution. Simulation module implements the elements of queuing rules in Queuing theory, and the system adopts the rules of treatment service, including first come first serve, random service and priority service. Medical resource allocation module implements the elements of services in Queuing theory. Optimization module, based on the principle of optimization of medical resources, implements the optimal allocation of medical resources by scheduling health care resource allocation and comparing the simulation results before scheduling.

\section{$B$. The patient generation module}

This module, based on elements of the input process of Queuing theory model, generates the patient's arrival time according to the input model parameters. According to the number of patients and model formula, it can calculate the number of patients generated each time, and the generated patients will be associated with a unique identifier. This module uses random number genration fucntion to produces the required number of each type of patient.

\section{The allocation model}

This module is designed and implemented based on service elements of the queuing theory model. It completes the scheduling process within medical resource allocation, simulation and optimization process. Health care resources include health care, major medical equipment and other necessary resources in medical procedures.

\section{The simulation module}

This module is designed based on the queuing rule element of the queuing theory model. It achieves the simulation of the hospital medical process. When patients enter the hospital for treatment process, they need to wait in line for treatment if there is no available medical staff to treat them. Simulating the medical process needs to take a variety of queuing rules into consideration, including the first come first service principle, random service and priority service.

Health care system is a discrete event simulation system. The system state changes are caused by simulation events. In order to solve the medical simulation model, we use the object-oriented simulation method which is also called the next event method. The next event method means that we can determine the next event occurs (such as the patients' arrival, classification, surgery, leave, etc.) and update the system's state through the elapse of time. The interval between two consecutive events is taken as a step to analog the clock's forwarding.

During the simulation process of the medical care, the start time and end time of prodedure events and the start time of non-procedure events are sorted in an ascending order. When these events are sorted, a time queue of events is obtained. Since patients keep entering and leaving the queue, the queue of the simulation process is dynamically changing. During the system simulation the queue length of the medical project, the project's busy ratio and the treatment ratio of each project are calculated in real-time. The number of patients who complete treatment is calculated when the simulation ends.

\section{E. The optimization module}

A threshold for busy ratio and a threshold for queue length are set before the system optimization starts. According to the evaluation conditions, medical service items are sorted in the simulation process. Then we identify the medical items which has a value of busy ratio less than corresponding threshold or has a smaller queuing length than schedulable projects, and mark the remaining as optimizable projects. In optimization process, the medical resources are transferred from the schedulable projects to the optimizable ones. Then the simulation is re-performed, and optimized process is the one with the largest number of patients passed.

\section{EXPERIMENTAL RESULTS AND ANALYSIS}

A. The pattern of patients' generation is showed in Table.1.

The medical service configuration is shown in Table. 2 .

The result of the simulation is listed in Table.3.

The following is the real-time system information and simulation results which we get in the simulation process. The vertical axis in Figure 1 represents the various medical items of various service desks and the horizontal axis represents the number of people who have completed the medical project during the simulation. 
B. According to the scheme of the wounded patient generation and the instance of the service setting program in the last section, we set a different threshold for the busy ratio and a different threshold for queue length. After optimization, the results are shown in Table.4 and Figure. 2. C. As we can see from the experiment results, there is a substantial increase in the number of patients treated after optimization within the specific time interval. After the optimization of hospital medical resources competes, the average waiting time of patients is reduced, and the number of hospital patients treated to pass is increased.

\section{CONCLUSIONS}

This paper discusses the medical treatment simualtion from the queuing theory perspective. We design and implement a Hospital Medical Process Simulation and Optimization System. This system can simulate medical procedures for hospital and optimize resources according to the medical evaluation system. It improves the utilization of medical resources of the hospital, reduces the average waiting time for patients, and improves the number of hospital patients treated successfully.

\section{REFERENCES}

[1] Xinhuan Han, Mengqian Zhu, Jing Wu. Decision Analysis of Optimal Queuing Model in Hospital Management System. Nanjing. Journal of Mathematical Medicine,2008,PP16-P17

[2] PengLiu, Min Liu. Patient flow analysis in the field of health services. Chinese Hospital Management.,2004,PP57-58

[3] Qi Wang. Optimization of outpatient flow based on queuing theory and the theory of business process reengineering. PhD thesis.,2008

[4] Yong Liu, Decai Wang, Zhengchao Feng. Measuring the efficiency of outpatient procedures using queuing theory models. Chinese Journal of Hospital Administration. 2005,PP806-809

[5] Operations Kum. Operational Research [M]. Beijing: Tsinghua University Press, 1990

TABLE I. THE PATTERN OF PATIENTS’ GENERATION

\begin{tabular}{|l|l|}
\hline The total number of the patients & 300 persons \\
\hline Distribution of patients' arrival time & Normal distribution \\
\hline The number of people severely burned & 96 persons \\
\hline The number of people moderately burned & 65 persons \\
\hline The number of people who have abdominal injury & 29 persons \\
\hline The number of people who have some injuries & 38 persons \\
\hline The number of people whose chests are openly injured & 72 persons \\
\hline
\end{tabular}

TABLE II. THE MEDICAL CONFIGURATION

\begin{tabular}{|l|l|}
\hline The total number of the doctors & 20 \\
\hline The number of the nurses & 30 \\
\hline The total number of the health desk & 60 \\
\hline
\end{tabular}

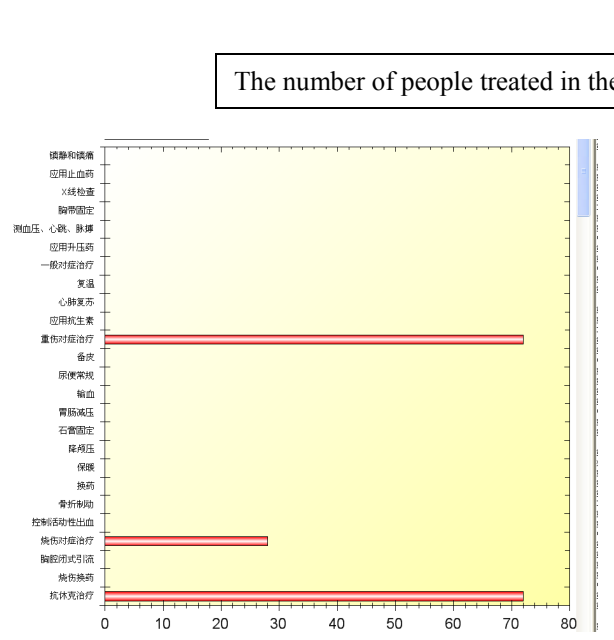

Figure 1. The number of people who complete of each medical project
TABLE III. THE RESULT

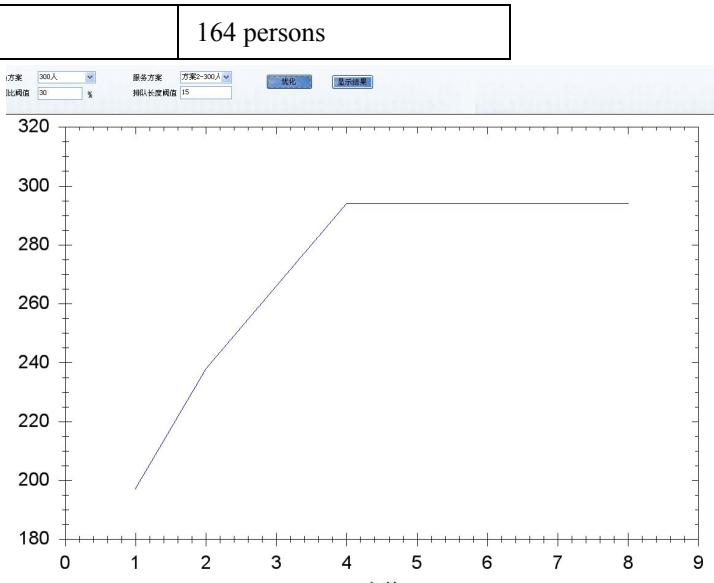

Figure 2. The process of optimization 
TABLE IV. THE OPTIMIZATION RESULT OF EACH SCHEME

\begin{tabular}{|l|l|l|l|}
\hline The scheme of optimization & The threshold for the percent of busy and spare time & The threshold for queue length & The number of people passed \\
\hline Scheme 1 & 20 & 30 & 252 \\
\hline Scheme 2 & 20 & 20 & 273 \\
\hline Scheme 3 & 20 & 30 & 294 \\
\hline Scheme 4 & 40 & 20 & 252 \\
\hline Scheme 5 & 40 & 10 & 273 \\
\hline Scheme 6 & 40 & 293 \\
\hline
\end{tabular}

